\title{
Effect of GEO satellite explosion on space environment
}

\author{
Hu Yiwen ${ }^{1, *}$, Zhang Zhanyue ${ }^{2}$, and Sun Hongqiang ${ }^{1}$ \\ ${ }^{1}$ Space Engineering University, Graduate School, 101416 Huairou, China \\ ${ }^{2}$ Space Engineering University, 101416 Huairou, China
}

\begin{abstract}
The distribution status of spatial targets in the geosynchronous orbit (GEO) is analysed, and the GEO orbital space environment model with space debris above $10 \mathrm{~cm}$ in feature size is established. The debris situation after the fragmentation of the satellite explosion in the orbit is simulated, and the safety of the space environment under the current conditions is analysed.
\end{abstract}

\section{Current status of GEO environment}

In April 2019, the Intelsat-29e satellite experienced a propellant leak in the GEO orbit and exploded finally, and the resulting debris caused serious hidden dangers to other satellites in the orbit. The GEO orbit is of great importance because of its synchronous nature and the orbit is a very limited resource. The height of the GEO orbit is about $35,786 \mathrm{~km}$. The atmosphere in the orbit is very thin, so there is almost no orbital attenuation in the GEO orbit. Therefore, once the explosion or collision occurs in the orbit, the generated debris will remain in the orbit for a long time, which would increase the risk of secondary collisions and even chain collisions ${ }^{[1]}$.

\subsection{Basic data of GEO}

The geostationary orbital protection area determined by the Inter-Agency Deformation Coordination Committee (IADC) is the area that the declination range of $\pm 15^{\circ}$, and the standard geostationary orbital height up and down $200 \mathrm{~km}$, i.e. $\mathrm{H}=35786 \pm 200 \mathrm{~km}$.

According to the public data of Space Surveillance Network (SSN), as of the 200th day of 2019, more than 18,000 on-orbit objects have been catalogued in space,

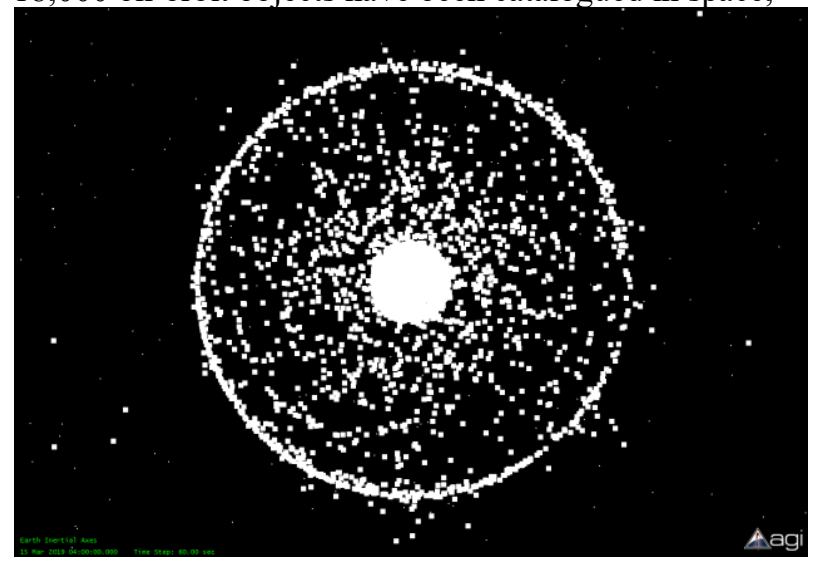

Fig. 1. Earth space top view.
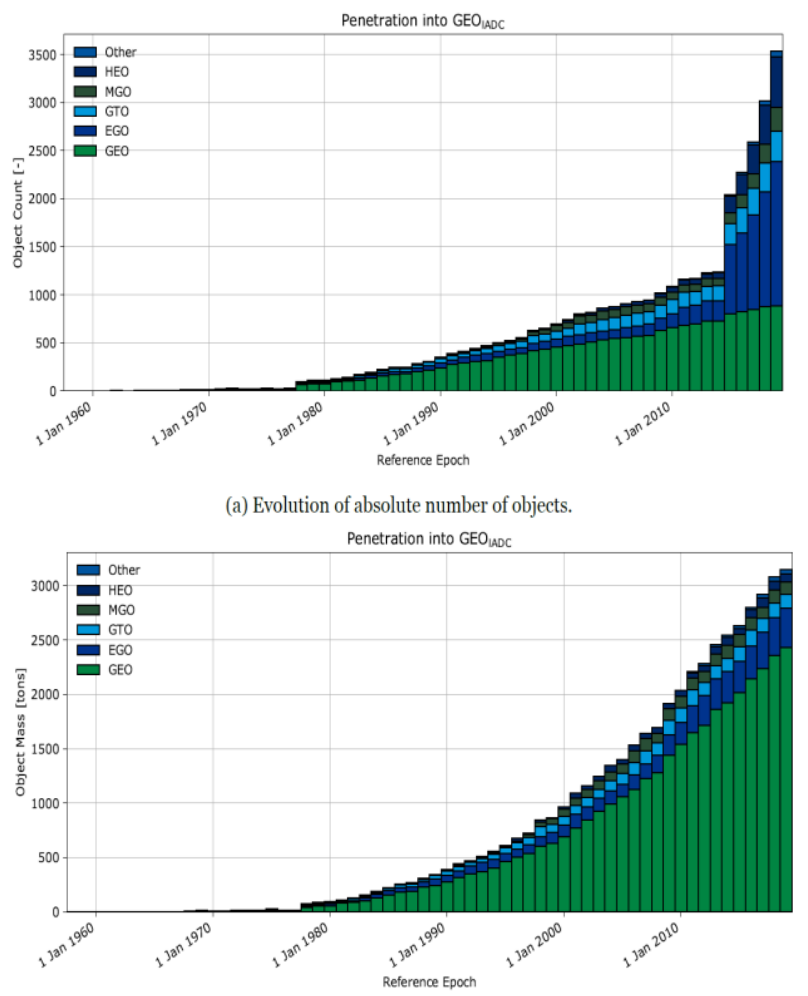

(b) Evolution of absolute mass.

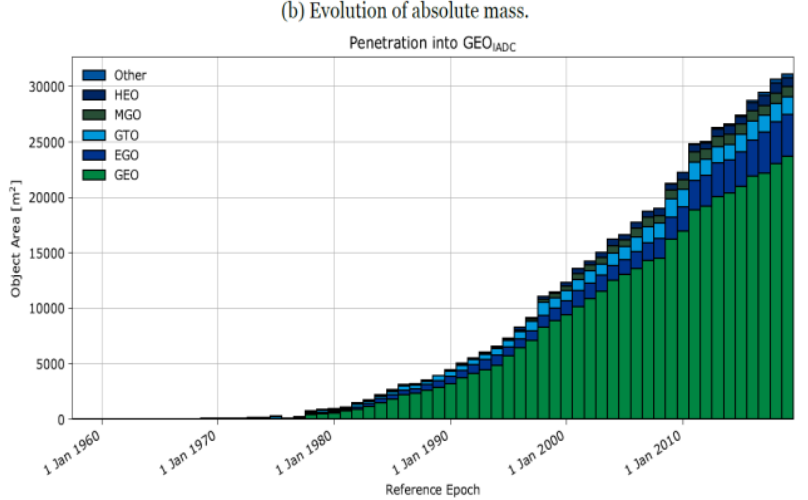

(c) Evolution of absolute area.

Fig. 2. Evolution of number of objects, mass and area in GEO.

* Corresponding author: huyw13@foxmail.com 
and their distribution in space is shown in the figure 1. ${ }^{[2]}$ Due to the limited capacity of the current space detection and surveillance system, the minimum detection size near the height of the geostationary orbit is about $1 \mathrm{~m}$. Over 1000 targets catalogued in the SSN would traverse or run in the GEO protection zone.

\subsection{Evolution in GEO}

Figure 2 shows the targets' evolution about the number, mass, and cross-sectional area in the GEO protection zone over time. ${ }^{[3]}$ EGO is the extended geostationary orbit, that is, the near-circular orbit whose eccentricity is less than 0.25 , orbital inclination is less than $25^{\circ}$, and semi-major axis is within $42164 \pm 4216 \mathrm{~km}$; GTO is the geosynchronous transfer orbit; $\mathrm{MGO}$ is the middlestationary crossing orbit that perigee is located in the middle earth orbit, and apogee is located in EGO; HEO is the high eccentric earth orbit, whose perigee is lower than EGO and apogee is higher than EGO.

It can be seen from the figure that the growth trend of the object mass and cross-sectional area in the GEO region was relatively stable, yet the growth of the target number in this region increased fiercely in the past five years.

Considering that explosive debris of the orbital targets adjacent to the GEO protected area would also affect the protected area, the GEO region in this article is defined as $10 \%$ up and down the standard semi-major axis of the geostationary orbit, that is, the area within $42164 \pm 4216 \mathrm{~km}$. Space objects that run or traverse in this orbital area are studied.

\subsection{Elements distribution in GEO}

By screening the TLE data published on Space-Track, the number of targets in the GEO region is about 2800. The distribution of the semi-major axis " $a$ " and the orbit period " $T$ " of these targets is shown in figure 3 and 4 . Most of the screened targets run on the orbit that $a \approx 42164 \mathrm{~km}, \quad T \approx 24 \mathrm{~h}$, that is, within the GEO region. What contributes to the peak located at $a \approx 26560 \mathrm{~km}$, $T \approx 12 \mathrm{~h}$ in the figures are mainly the "Molniya" communication satellites with apogee of about 40,000 $\mathrm{km}$ and perigee of about $400 \mathrm{~km}$. The peak at $a \approx 24500 \mathrm{~km}$ and $T \approx 10.5 \mathrm{~h}$ is due to the concentrated distribution of the fuel slag released by the solid rocket motor (SRM) on the geosynchronous transfer orbit (GTO).

The distribution of orbital inclination " $i$ " and eccentricity " $e$ " of spatial targets in the GEO region is shown in figure 5 and 6 . The inclination of most targets is between 0 and $15^{\circ}$, which is mainly caused by the sun and moon gravity and the non-spherical perturbation of the Earth. The eccentricity is mostly less than 0.005. The peak located at $i \approx 63.4^{\circ}$ in the orbital inclination distribution is related to the "Molniya" satellite. The eccentricity of the GTO orbit is about 0.73 , and the eccentricity of the "Molniya" orbit is about 0.74 , which leads to the peak at $e \approx 0.735$ in figure $5^{[4]}$.

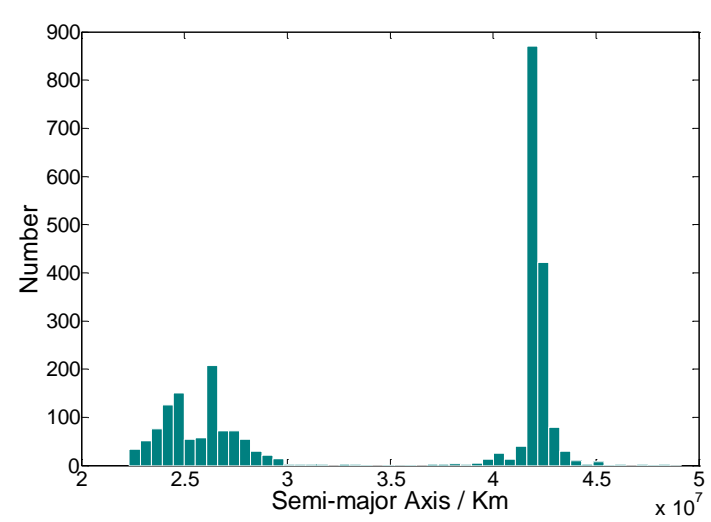

Fig. 3. Distribution of the semi-major axis.

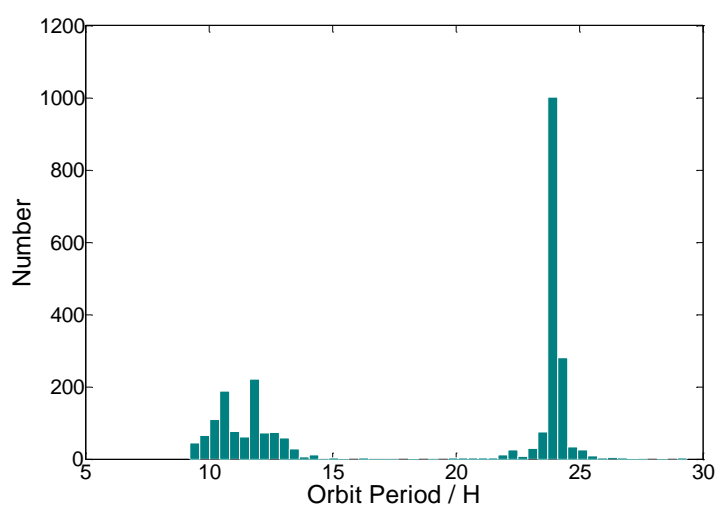

Fig. 4. Distribution of orbit period.

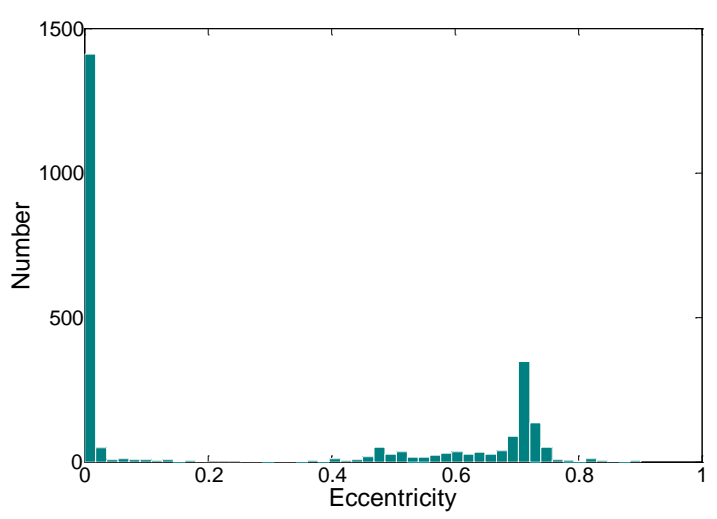

Fig. 5. Distribution of orbit eccentricity.

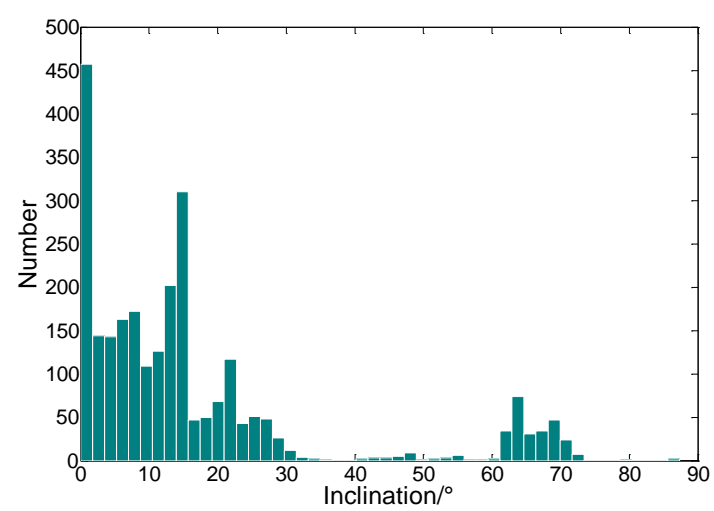

Fig. 6. Distribution of orbit inclination. 


\section{GEO Spatial data correction}

Limited by the observation conditions, the current SSN catalogue data can not cover the full-size space target, especially for objects smaller than $1 \mathrm{~m}$ in the GEO area, so the catalogue data of the GEO needs to be corrected and supplemented.

For data that SSN does not include currently, it can be estimated by the empirical calibration factor $c_{T L E}{ }^{[4]}$

$$
\mathrm{c}_{T L E}=10^{\frac{1}{2} \exp \left\{-2.464\left[\lg \left(d_{S S N}\right)+1.22\right]^{2}\right\}}
$$

The value of the SSN detection capability $d_{S S N}$ is determined by the following empirical formula, whose unit is $m$ :

$$
\mathrm{d}_{S S N}(H)=\left\{\begin{array}{cc}
0.089, & H \leq 620 \mathrm{~km} \\
10^{-2.727+0.604 \lg H}, & 620<H \leq 1300 \mathrm{~km} \\
10^{-6.517+1.819 \lg H}, & 1300<H \leq 3800 \mathrm{~km} \\
10, & H>3800 \mathrm{~km}
\end{array}\right.
$$

It is estimated by the above formula that the number of objects larger than $1 \mathrm{~m}$ in the GEO protection area is about 1000, the number of targets with a size between 0.1 and $1 \mathrm{~m}$ is about 2,200, and the number of debris whose size larger than $0.01 \mathrm{~m}$ exceeds 64000 .

According to the orbital elements of the known GEO targets with the size larger than $1 \mathrm{~m}$, the statistical rule of the distribution is obtained. Then the probability density function of each orbital element is generated by multipeak Gaussian fitting together with polynomial fitting, and the orbital elements of the uncatalogued debris are generated accordingly.

\section{Fragmentation Model}

The NASA standard fragmentation model can be divided into two parts: explosion fragmentation and collision fragmentation. This article only considers the situations where initial fragmentation condition is an explosion ${ }^{[4]}$.

\subsection{Fragmentation debris size distribution}

First, the feature size $L_{c}$ is defined as the average value of the sizes in three axis:

$$
L_{c}=\left(L_{x}+L_{y}+L_{z}\right) / 3
$$

Cumulative amount distributed of explosion debris whose feature size is greater than a certain $L_{c}$ :

$$
N\left(L_{c}\right)=6 \mathrm{c}_{s} L_{c}^{-1.6}
$$

Where $c_{s}$ is the scaling parameter associated with the type of explosion, and the value is between 0 and 1 .

\subsection{Area to mass ratio distribution}

For debris of some feature size, its area-mass ratio parameter and feature size parameter conform to:

$$
\begin{gathered}
\chi=\lg (A / M) \\
\theta=\lg \left(L_{c}\right) \\
p(\chi, \theta)=\alpha(\theta) N_{1}(\chi)+[1-\alpha(\theta)] N_{2}(\chi)
\end{gathered}
$$

Therein $N_{i}(i=1,2)$ is a normal distribution, and its probability density function is:

$$
N_{\mathrm{i}}(\chi)=\frac{1}{\sigma_{i} \sqrt{2 \pi}} \exp \left[-\frac{\left(\chi-\mu_{i}\right)^{2}}{2 \sigma_{i}^{2}}\right]
$$

For explosive debris with feature sizes greater than $11 \mathrm{~cm}$, the relevant parameters take values:

$$
\begin{gathered}
\alpha(\theta)=\left\{\begin{array}{lc}
1, & \theta \leq-1.4 \\
1-0.357(\theta+1.4), & -1.4<\theta<0 \\
0.5, & \theta \geq 0
\end{array}\right. \\
\mu_{1}(\theta)=\left\{\begin{array}{lc}
-0.45, & \theta \leq-0.5 \\
-0.45-0.9(\theta+0.5), & -0.5<\theta<0 \\
-0.9, & \theta \geq 0
\end{array}\right. \\
\sigma_{1}(\theta)=0.55 \\
\mu_{2}(\theta)=-0.9 \\
\begin{array}{ll}
0.28, & \theta \leq-1.95 \\
0.28-0.1636(\theta+1), & -1.95<\theta<0.55 \\
0.1, & \theta \geq 0.55
\end{array}
\end{gathered}
$$

For explosive debris with a feature size less than 1.7 $\mathrm{cm}$, take $\alpha(\theta)=1$, the area to mass ratio becomes a single parameter form, and the parameter values are:

$$
\begin{gathered}
\mu_{1}(\theta)=\left\{\begin{array}{lc}
-0.3, & \theta \leq-1.75 \\
-0.3-1.4(\theta+1.75), & -1.75<\theta<-1.25 \\
-1.0, & \theta \geq-1.25
\end{array}\right. \\
\sigma_{1}(\theta)= \begin{cases}0.2, & \theta<-3.5 \\
0.2+1.333(\theta+3.5), & \theta \geq-3.5\end{cases}
\end{gathered}
$$

For the debris between $1.7 \mathrm{~cm}$ and $11 \mathrm{~cm}$, the values can be obtained by comparing the size of $\tilde{\zeta}\left(L_{c}\right)$ and the random number $\zeta \in[0,1]$. While $\zeta>\tilde{\zeta}\left(L_{c}\right)$, use the twoparameter probability density function which is suitable for the larger size, to obtain the area-mass ratio distribution. A single parameter distribution for smaller sizes was used while $\zeta<\tilde{\zeta}\left(L_{c}\right)$. Therein:

$$
\tilde{\zeta}\left(L_{c}\right)=10\left(\lg L_{c}+1.76\right)
$$

The cross-sectional area " $A$ " of debris can be obtained from the feature size, and the relationship between $A$ and $L_{c}$ is: 


$$
A= \begin{cases}0.540424 L_{c}^{2}, & L_{c}<0.00167 m \\ 0.556945 L_{c}^{2.0047077}, & L_{c} \geq 0.00167 m\end{cases}
$$

Therefore, the mass of debris can be obtained by dividing the cross-sectional area by the area-mass ratio:

$$
\mathrm{m}=\frac{\mathrm{A}}{\mathrm{A} / \mathrm{m}}
$$

\subsection{Separation velocity distribution}

The separation velocity direction of the generated debris is an omnidirectional uniform distribution, and the $\operatorname{logarithm} \delta=\lg (\Delta v)$ of the separation velocity increment conform to:

$$
p(\delta)=\frac{1}{\sigma_{v} \sqrt{2 \pi}} \exp \left[-\frac{\left(\chi-\mu_{v}\right)^{2}}{2 \sigma_{v}^{2}}\right]
$$

where $\mu_{v}=0.9 \chi+2.90, \sigma_{v}=0.4$.

According to the nature of the single parameter normal distribution, the probability density $p(v)$ is integrated to obtain the probability $P_{v}$ and therefore:

$$
\Delta v=10^{\wedge}\left[\mu_{v}+\sigma_{v} \sqrt{2} \operatorname{erf}^{-1}\left(2 P_{v}-1\right)\right]
$$

The explosion produces 9497 space debris larger than $0.01 \mathrm{~m}$. Figure 9 shows the distribution of the apogee and perigee of the explosive debris along with its period. It can be seen from the figure that the orbital period of most fragments is still around $24 \mathrm{~h}$, indicating that the $\Delta v$ obtained by these debris has less impact; The left side of the intersection in the figure represents the debris with a period of less than $24 \mathrm{~h}$, because the obtained " $-\Delta v$ " reduces the orbital perigee, thus shortening the orbital period; and the right side of the intersection represents the fragment with a longer period, because " $+\Delta v$ " raises the apogee ${ }^{[4]}$.

On the whole, the debris is distributed in an " $\mathrm{X}$ " shape. It is seen from the figure, since the original orbital target was running on a near-circular orbit before the explosion, the explosion point would become a perigee or apogee of the explosive debris orbit, that is, neither the perigee or the apogee of the debris is still near $35,786 \mathrm{~km}$.

\section{Conclusion}

For these explosive debris, the time and location of their crossing through the GEO protection zone cannot be predicted in a short time, thus the explosive debris is easy to cause chain collisions in the short term. Furthermore, because the GEO region lacks atmospheric perturbation, the debris would survive hundreds of years and bring about the long-term threatens to GEO region.

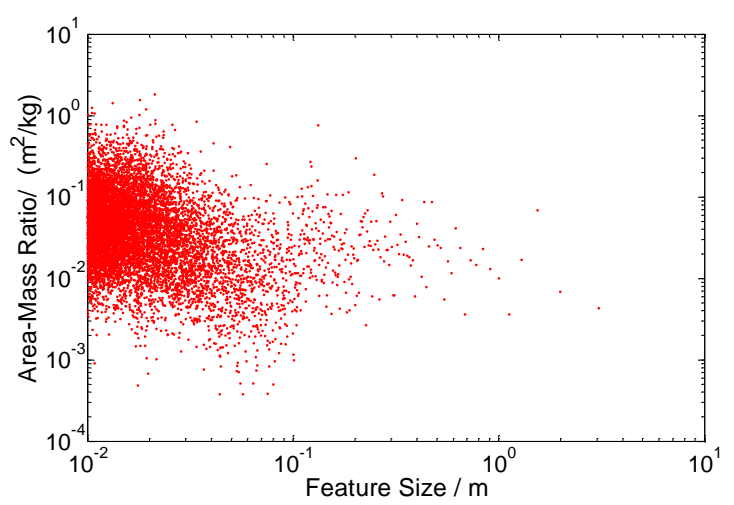

Fig.7. Distribution of area-mass along with feature size.

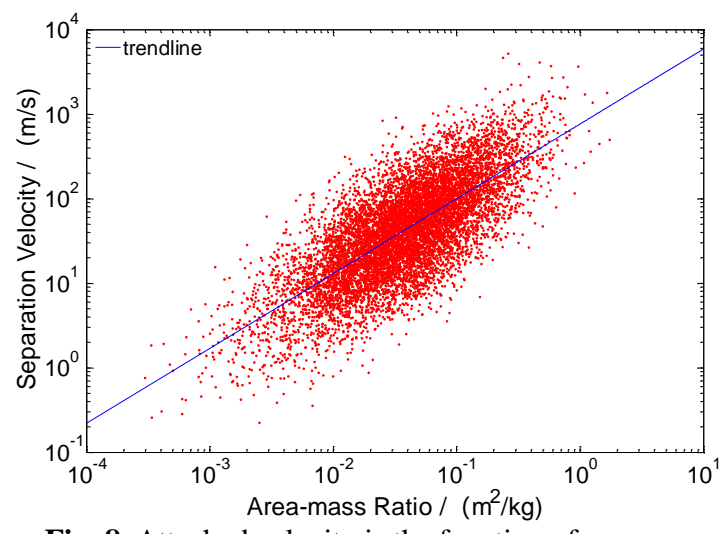

Fig. 8. Attached velocity is the function of area-mass.

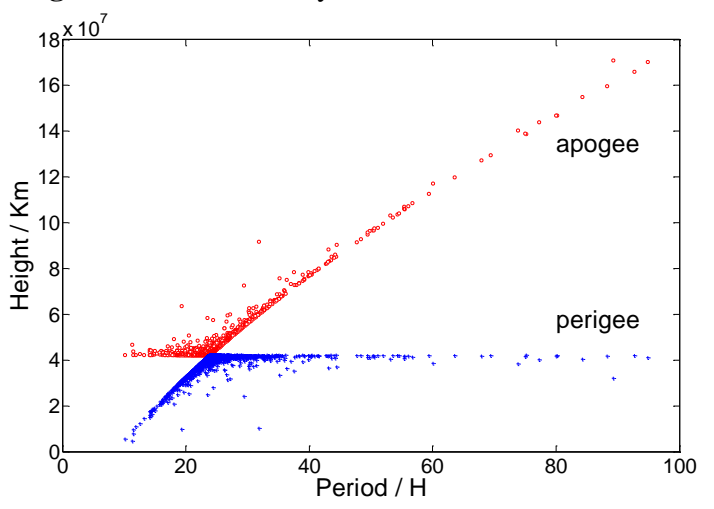

Fig. 9. Distribution of apogee and perigee along with period.

\section{References}

1. D.S. McKnight, F.R. Di Pentino, New insights on the orbital debris collision hazard at GEO, Acta Astronautica 85(2013)73-82.

2. Space Surveillance Data Available From Joint Space Operations Center, 20 July 2019, (http://www.space-track.org).

3. ESA's Annual Space Environment Report 2019, ESA Space Debris Office, 2 May 2019.

4. Heiner Klinkrad, Space debris: models and risk analysis, Springer, 2006. 